\title{
Fractal to Euclidean crossover and scalling for random walks on percolation clusters. II. Three-dimensional lattices ${ }^{a}$
}

\author{
Panos Argyrakis \\ Department of Physics, University of Crete, Iraklion, Crete, Greece \\ Raoul Kopelman \\ Department of Chemistry, University of Michigan, Ann Arbor, Michigan, 48109-1055
}

(Received 26 April 1985; accepted 30 May 1985)

\begin{abstract}
We perform random walk simulations on binary three-dimensional simple cubic lattices covering the entire ratio of open/closed sites (fraction $p$ ) from the critical percolation threshold to the perfect crystal. We observe fractal behavior at the critical point and derive the value of the number-of-sites-visited exponent, in excellent agreement with previous work or conjectures, but with a new and improved computational algorithm that extends the calculation to the long time limit. We show the crossover to the classical Euclidean behavior in these lattices and discuss its onset as a function of the fraction $p$. We compare the observed trends with the two-dimensional case.
\end{abstract}

\section{INTRODUCTION}

In a recent paper $^{1}$ (referred to as paper I) we examined the behavior of random walks on percolation clusters in two dimensions, a good test case for fractal behavior both in the static (size and shape of clusters) and in the dynamic (diffusion of a particle) sense. We first investigated the case of the critical percolation threshold, where we found the value of the spectral dimension $(\approx 4 / 3)$ and verified the validity of the relation involving the Hausdorff dimension $\approx 1.9$. We then covered the region around the threshold where we found and verified the validity of the universal critical exponents. Subsequently we covered the entire regime from the critical point to the perfect lattice limit, establishing the crossovers from the fractal to the Euclidean limit. This was done, using simulation data, by monitoring several random walk properties as a function of time. ${ }^{2-4}$ Our data has the advantage first of using relatively large lattices and second of extending to the long time limit. In the present paper we extend these ideas to three-dimensional lattices. The renewed interest in this case stems from the fact that the $2 \mathrm{D}$ and $3 \mathrm{D}$ random walks differ in their behavior. In fact, the $3 D$ lattice is a simpler case, because here $\left\langle S_{N}\right\rangle$, the average number of distinct sites visited in an $N$-step walk, is simply

$$
\left\langle S_{N}\right\rangle=c \cdot N, \text { three-dimensional, } N \rightarrow \infty
$$

while for the two-dimensional case,

$$
\left\langle S_{N}\right\rangle=c N / \log N, \text { two-dimensional square lattice. }
$$

Here $c$ is a constant dependent on the lattice topology. We see that $\left\langle S_{N}\right\rangle$ is directly proportional to $N$ (time) for the $3 D$

\footnotetext{
"Supported by NATO grant No. SA 5205 RG 295/82 and NSF grant DMR 8303919.
}

case, but has the more complex $N / \log N$ dependence for the 2D case. Therefore, the crossovers may be expected to be different.

For percolating clusters the average number of distinct sites visited $\left\langle S_{N}\right\rangle$ in an $N$-step walk has an asymptotic form independent of the lattice topology, given by

$$
\left\langle S_{N}\right\rangle \sim N^{d / 2}, \quad N \rightarrow \infty,
$$

where $d_{s}$ is the spectral dimension ${ }^{5,6}$ with a value in the vicinity of $4 / 3$. There has been a pronounced interest ${ }^{9}$ in the exact value of $d_{s}$, and the most recent calculations ${ }^{2}$ indeed

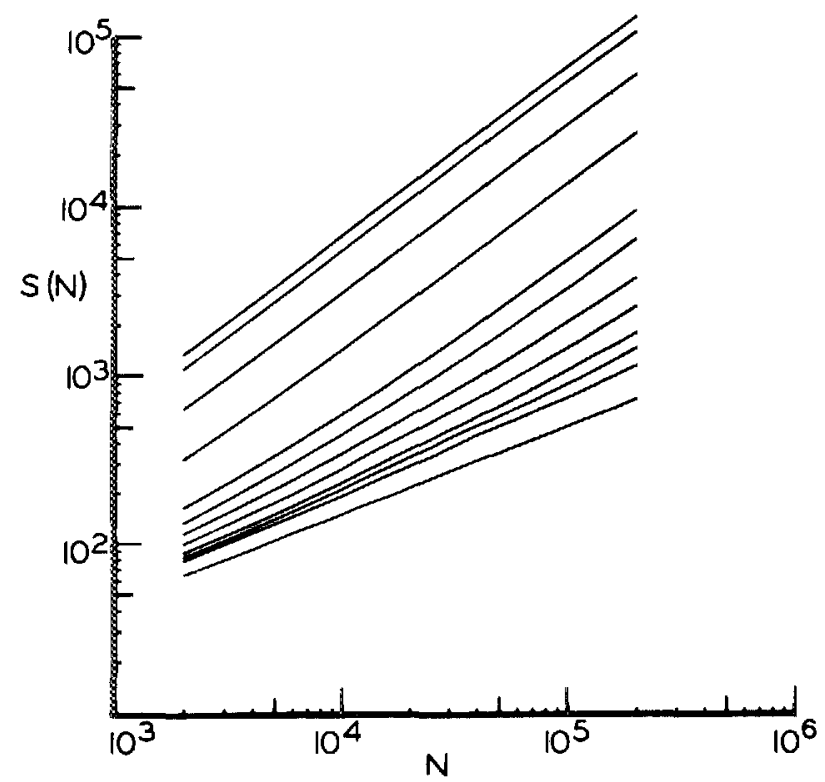

FIG. 1. $S_{N}$ vs $N$ for binary three-dimensional simple cubic lattices. Random walk may originate on any cluster. Top to bottom: $p=1.00,0.75,0.50,0.40$, $0.35,0.34,0.33,0.325,0.32,0.3175,0.315,0.3117$. 
show that its exact value in two dimensions is $2 \%-3 \%$ lower than the originally proposed value of $4 / 3$. The scaling relation (see paper I) is

$$
S_{N}(t \rightarrow \infty, p)=t^{d / 2} f\left[\left(\frac{p}{p_{c}}-1\right) t^{1 /(2 v-\beta+\mu)}\right]
$$

with the crossover time $t^{*}$ being

$$
t^{*} \sim\left|p-p_{c}\right|^{-(2 v-\beta+\mu)} \text {. }
$$

We now use the three-dimensional values of the exponents $^{8} v$ (correlation length), $\beta$ (the percolation probability), and $\mu$ (conductivity exponent). We also investigate the range of the effective $f$ values for the $p$ range $p=0.31$ (slightly below $p_{c}$ ) to $p=1.00$ (a perfect lattice), defined by $S_{N} \sim N^{f}$.

\section{METHOD OF CALCULATIONS}

We used the recentiy improved ${ }^{1}$ algorithm for Monte Carlo simulation techniques to monitor several random walk properties on three-dimensional lattices. All details are given in paper I. The size of the lattice used is $160 \times 160 \times 160$ ( $=4 \times 10^{6}$ sites) with a maximum value of $N$ of 200000 steps. All lattices are generated using the cluster growth technique; therefore random walks may originate on any-size cluster, and not just on the infinite percolating cluster. Using this method only one random walk realization is executed on each lattice. Most of the calculations were performed with the DEC random number generator RAN. Also, our results were compared to the expected values in the perfect lattice limit to ensure that the random walk proceeds properly and we find excellent agreement.

\section{脽. RESULTS AND DISCUSSION}

For the three-dimensional simple cubic lattice the critical percolation threshold is ${ }^{8} p_{c}=0.3117$. We include results that are averages of 1000 realizations on the following lattices: $p=0.3117,0.315,0.3175,0.32,0.325,0.33,0.34,0.35$, $0.4,0.5,0.75,1.00$. Figure 1 shows the crossover for the simple cubic lattice site percolation case. Using the equation $S_{N} \propto N^{f}$ we see that $\log S_{N}$ is linear with $\log N$, while the slope of the appropriate curve gives the $f$ exponent. From the $p_{c}=0.3117$ curve we derive a slope of $f=0.53$, giving $d_{s}^{\prime}$ $=1.06$, which is the exponent that describes random walks on all size clusters. Assuming a $d_{s}=4 / 3$ for random walks on the largest percolating cluster one gets ${ }^{10}\left(d-\beta / v=d_{H}\right.$, the Hausdorff dimension),

$$
d_{s}^{\prime}=d_{s}\left[2-\frac{d}{d-\beta / v}\right]=1.07
$$

where $d$ is the Euclidean dimensionality; here $d=3$, $\beta=0.44$, and $v=0.88$ for the simple cubic lattice. ${ }^{8}$ Thus, these simulation results are in excellent agreement with the reported $^{5,6}$ predictions for the three-dimensional lattices. We note that the "theoretical" value $d_{s}^{\prime}=1.07$ has an uncertainty of up to $2 \%$ due to the uncertainties in the critical exponents. Thus we cannot test the value of $d_{s}=4 / 3$ to the same precision we did for the $2 \mathrm{D}$ case (I). We also note that for the size of lattice used in this study ( $L=4 \times 10^{6}$ sites) our

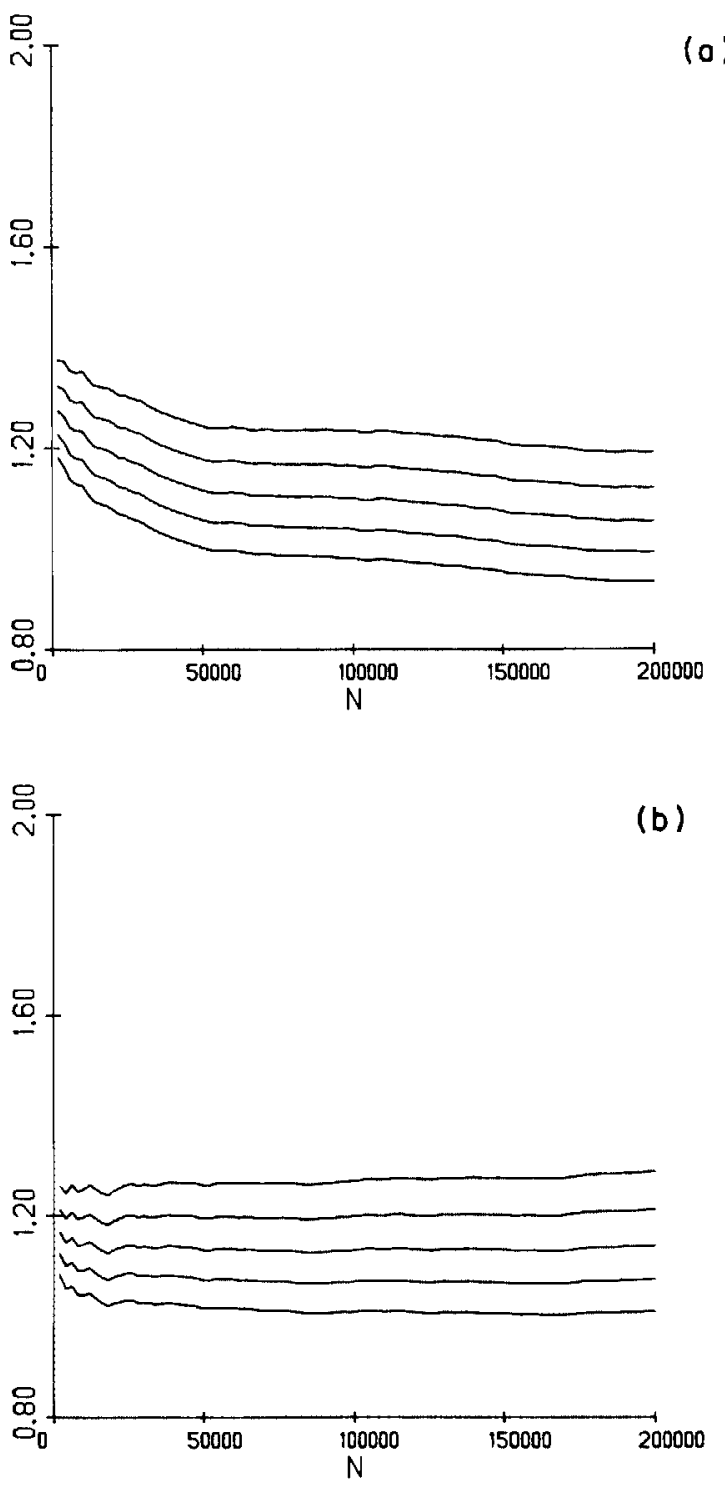

(o)

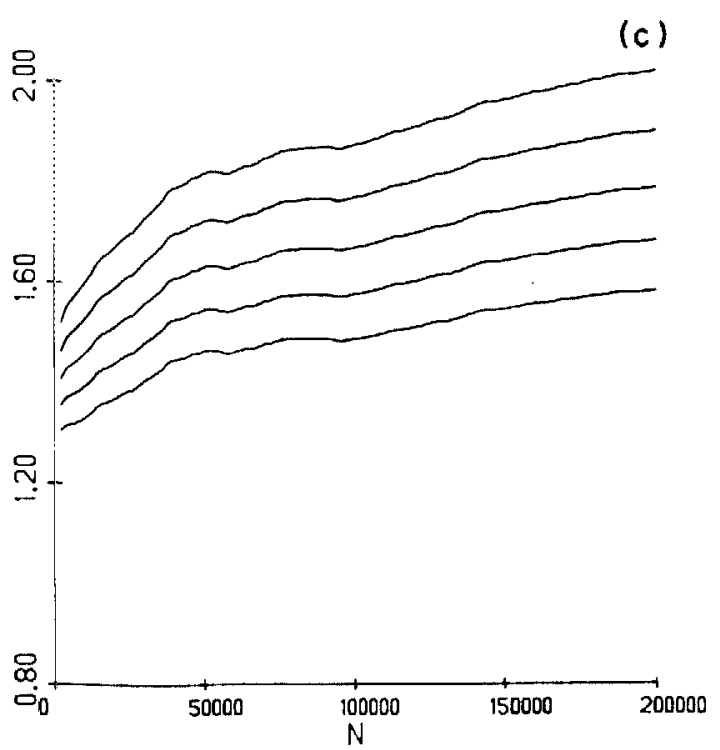

FIG. 2. Plot of $S_{N} / N^{d ; / 2}$ vs $N$ with the $d_{s}^{\prime}$ exponent being an adjustable parameter. Here $d ;=1.04,1.05,1.06,1.07$, and 1.08 (top to bottom). Random walks on any-size cluster at: (a) $p=0.31$, (b) $p=0.3117$, and (c) $p=0.315$. 


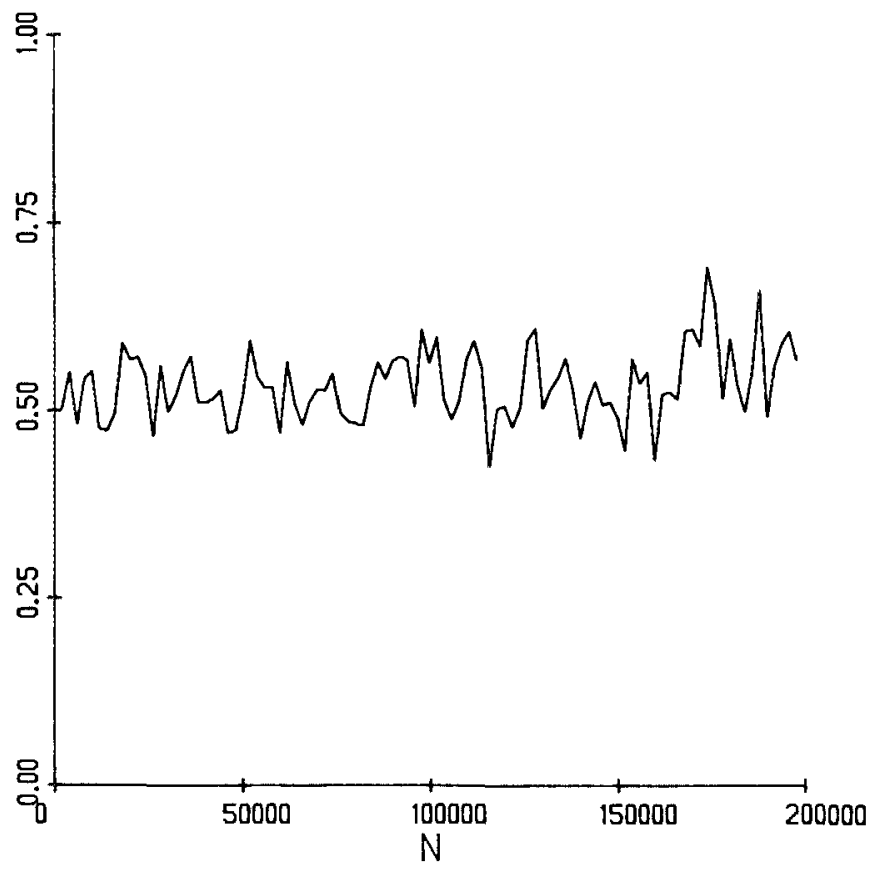

FIG.3. Plot of $d\left(\log S_{N} V d(\log N)\right.$ vs $N$ for random walks on any-sizecluster at $p=0.3117$.

results are free from any finite size caused false revisitation corrections for $N$ up to approximately $N=1.5 \times 10^{5}$ steps.

The $p=1.00$ curve gives an $f$ value of $f=0.99$, very close to the asymptotic value of 1.0. For all intermediate values of $p$ the lines are again curved upwards (see paper I) showing the crossover from fractal to Euclidean behavior.

In Fig. 2 we plot $S / N^{d_{s}^{\prime}}$ vs $N$ for random walks at three

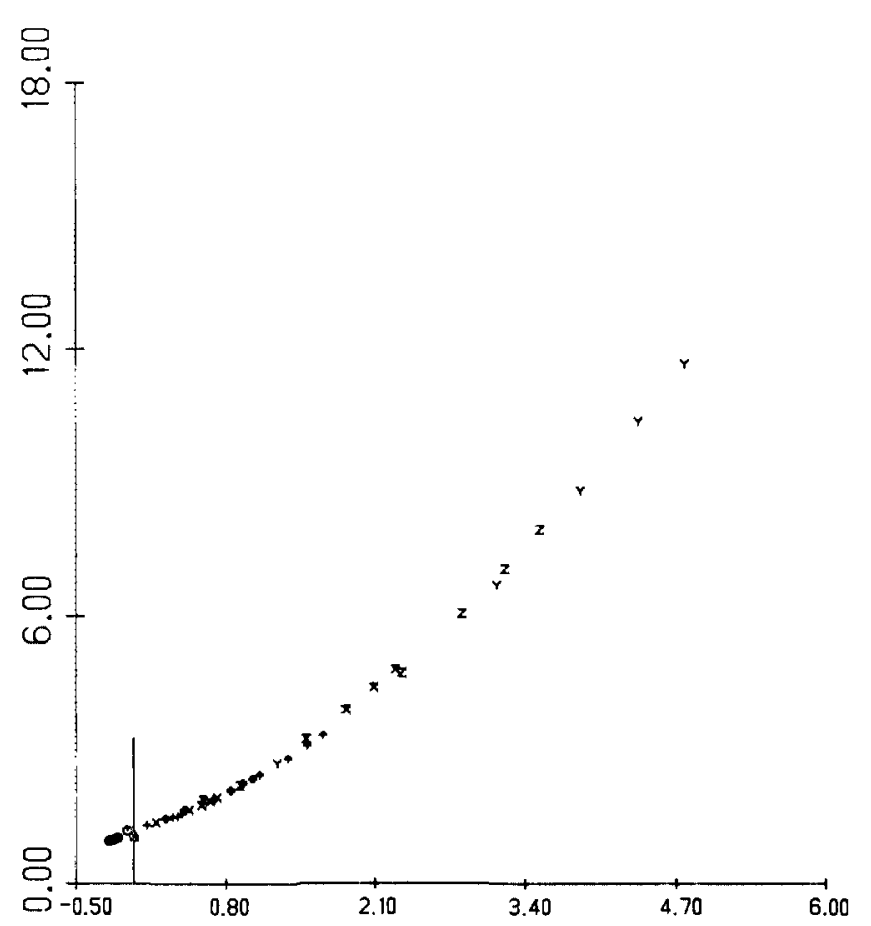

FIG. 4. Scaling plot $S_{N} / N^{d ; / 2}$ vs $\left(p / p_{c}-1\right) N^{0.303}$ for walks on any-size cluster. Here $d_{s}=1.06$. The different symbols correspond to different $p$ values: $p=0.31,0.3117,0.315,0.3175,0.32,0.33,0.34$, and 0.35 . Note that the $p=0.31$ values are below the threshold and, thus, appear to the left of the line $x=0$. The $N$ values shown are: $2000,42000,82000,122000$, and 162000 . different lattice concentrations: (a) $p=0.31$, slightly below $p_{c}$; (b) at $p_{c}=0.3117$ exactly; and (c) at $p=0.315$, slightly above $p_{c}$. In each case we plot five different values of $d_{s}^{\prime}$, $d_{s}^{\prime}=1.04,1.05,1.06,1.07$, and 1.08 in order to see its variation. In this plot the $y$ axis is really the proportionality constant in Eq. (3) and we observe that only in the $p_{c}=0.3117$ case does it give a fairly constant value while at $p>p_{c}$ the curves give an increasing value for this constant and at $p<p_{c}$ they produce a decreasing value. Simply explained, in the first case we again (see paper I) have a manifestation of the crossover to high $p$ concentration, while in the latter case we see the first evidence for the "saturation" of the finite clusters " ", the problem of random walks on a "dust." The important point to notice in these figures is that using our new algorithm even extremely small deviations from criticality show these trends. In addition, we again get the best agreement for $d_{s}^{\prime}=1.07$, within about $2 \%$ uncertainty. As a further test in Fig. 3 we plot $d\left(\log S_{N}\right) / d(\log N)$ vs $N$ and as expected we get a straight line but with a high random noise. This noise is inherent in the differential way of producing this plot but what is important here is not the local fluctuation but the overall straight horizontal line which on the $y$ axis has a value of $f=0.53$, i.e., twice this value gives again the $d_{s}=1.06$ exponent.

The scaling of data is given in Figs. 4 and 5 . Using Eq. (4) we set $d_{s}^{\prime}=1.06, v=0.88, \quad \beta=0.44$,

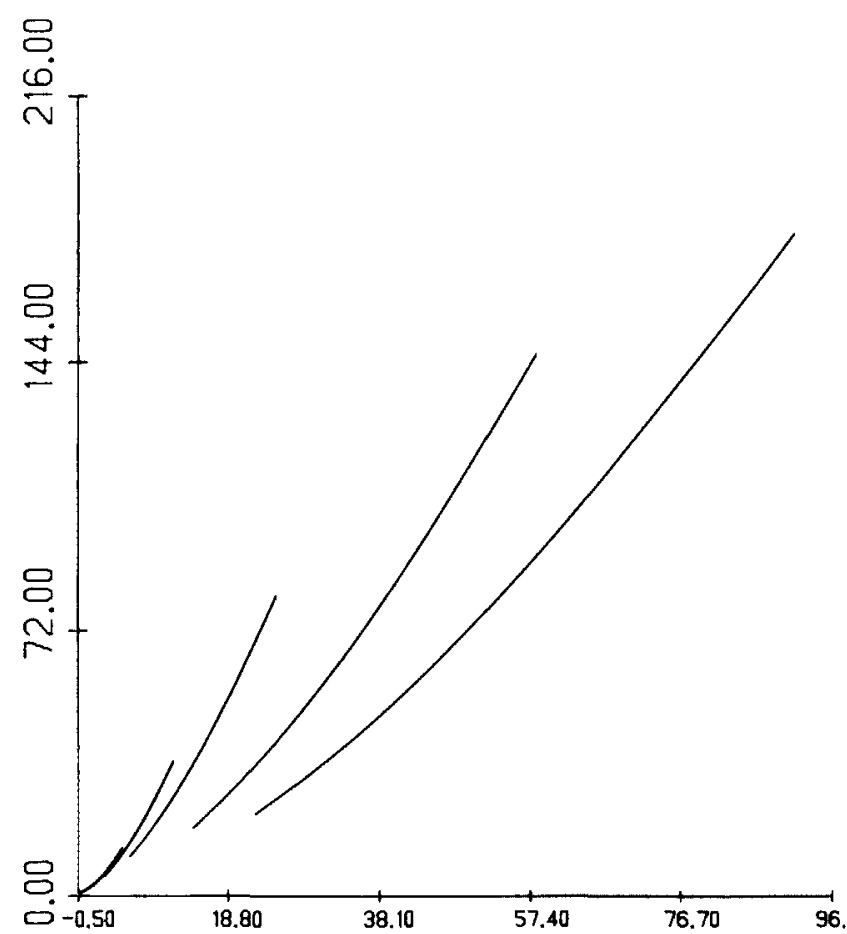

FIG. 5. Extended scaling plot: $S_{N} / N^{d: / 2}$ vs $\left(p / p_{c}-1\right) N^{0.303}$. Same as Fig. 3 (contracted) but with the addition of curves for $p=0.4,0.5,0.75$, and 1.00 (left to right). Note that the $p=0.4$ curve "merges" with the lower $p$ scaling curves of Fig. 4. However, the $p=0.5,0.75$, and 1.0 curves seem to deviate from it. 


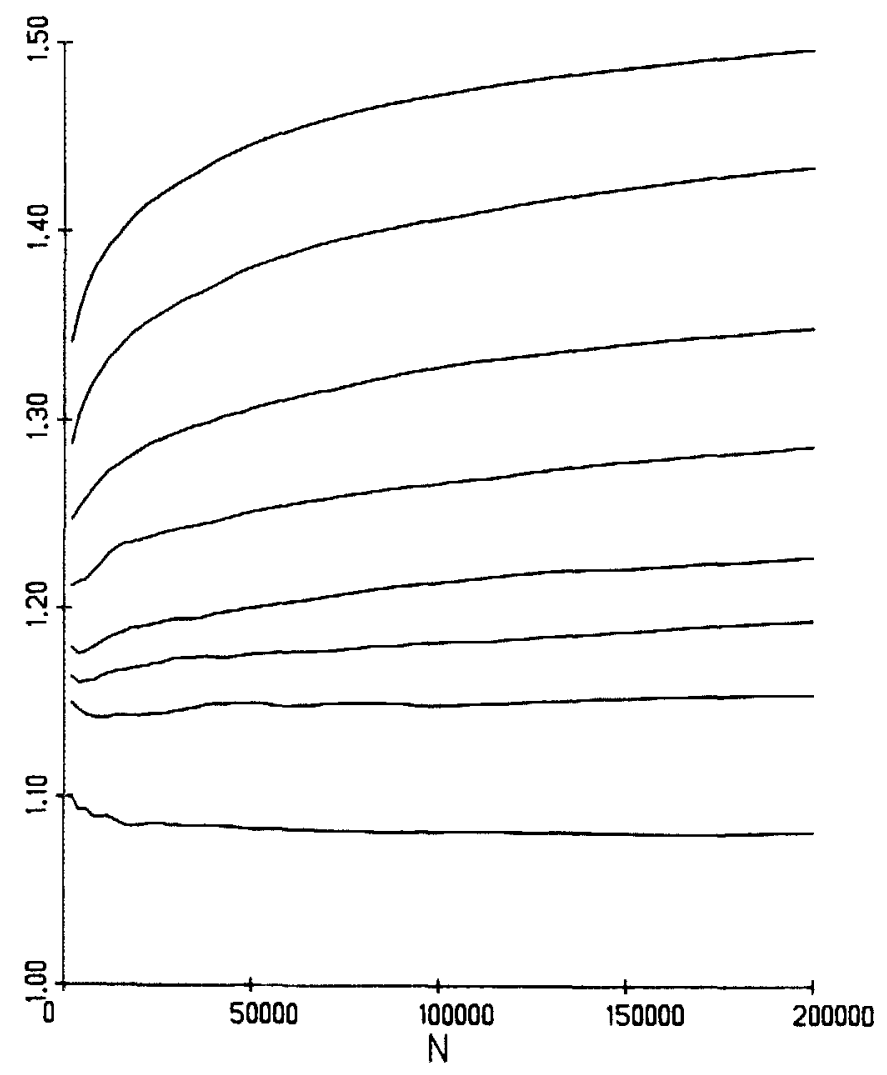

FIG. 6. Plot of $2 \log S_{N} / \log N$ vs $N$ for random walks originating on any cluster. Bottom to top: $p=0.3117,0.315,0.3175,0.32,0.325,0.33,0.34$, and 0.35 .

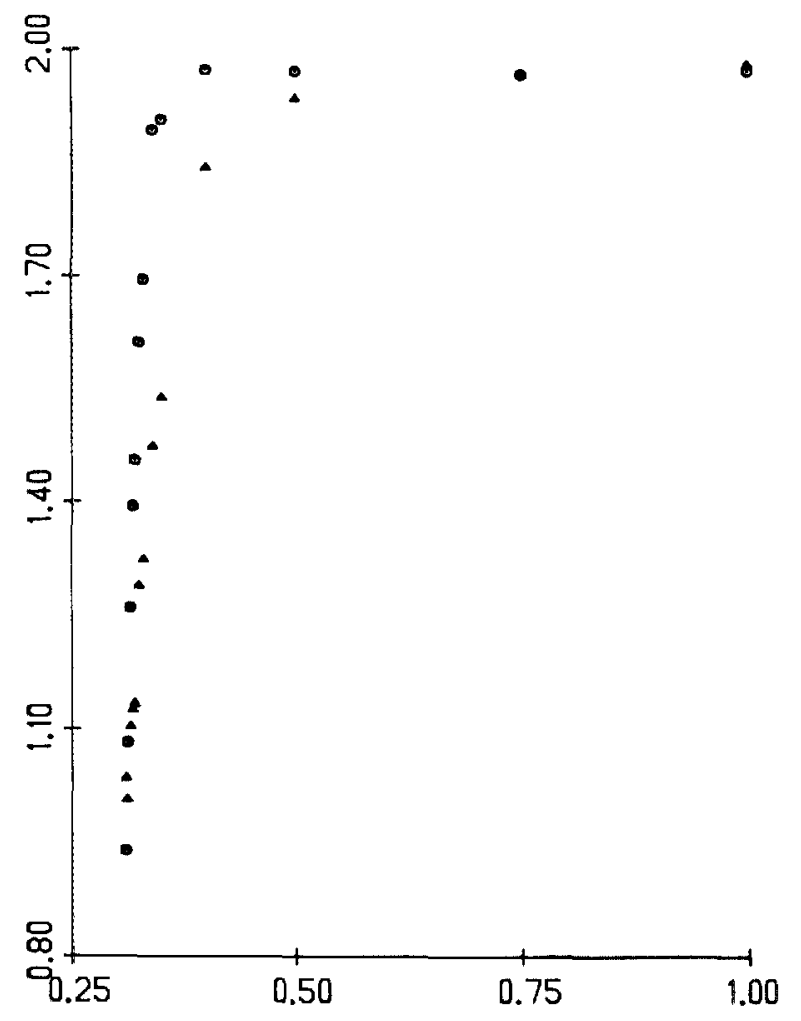

FIG. 7. Plot of $2 f v s p$ for walks originating on any-size cluster. Squaresfrom long time limit $(N=200000$ over $N=100000)$. Triangles-from short time limit $(N=4000$ over $N=2000)$. $\mu=(1 / 2)[v(3 d-4)-\beta]=1.98$. Kence, $2 v-\beta+\mu=3.3$. In Fig. 4 many different values of $p$ and $N$ are used to produce a scaling curve quite satisfactory. In Fig. 5 we see that scaling is valid only up to $p=0.35$ while scaling breaks down for $p=0.40$ and above. Therefore the idea of scaling and the validity of the correlation length $\xi$ and the critical exponents $v$ and $\beta$ are limited to the interval $0.3117<p<0.35$. Thus, these two figures provide us both with a verification of the universality hypothesis around the critical point and the region of validity of this universality.

Figure 6 gives us the value of the effective $d_{s}^{\prime}$ exponent for a wide $p$ range. The $p=0.3117$ curves are almost straight (horizontal), as expected, while the higher $p$ curves give us the crossover in the direction of the Euclidean behavior. Values of up to $p=0.35$ are used in these curves, since above $p=0.35$ all curves show a sharply increasing $d_{s}$ exponent.

In Fig. 7 we show the behavior of $2 f$ as a function of $p$ for two different limits, the early time limit ( $N=2000$ steps) and long-time behavior $\left(N=2 \times 10^{5}\right.$ steps). First we notice that $2 f$ increases rather fast as $p$ increases from the critical point $p_{c}=0.3117$. The curves remind us of the sharp rise in the "percolation-type" curves for the formation of the largest cluster at the critical point. This trend is only a manifestation of the earlier observed behavior that the fractal region is rather narrow close to $p_{c}$ and one approaches rather quickly the classical behavior. We also notice that the rise in the $2 f$ value is much sharper at longer times that at short time, an observation similar to that for the two-dimensional lattices (see Fig. 6 of paper 1).

Concluding, we presented long time randorn walk simulations on simple-cubic lattices exactly at the critical percolation threshold and above it, up to the perfect crystal. Our results confirm the scaling, crossover, and universality hypothesis of random walks on fractal structures. Furthermore, we were able to define the region of validity of the above assumptions and show in detail the transition to the classical behavior.

'P. Argyrakis and R. Kopelman, J. Chem. Phys. 81, 1015 (1984).

${ }^{2}$ P. Argyrakis, L. W. Anacker, and R. Kopelman, J. Stat. Phys. 36, 579 (1984).

${ }^{3}$ J. S. Newhouse, P. Argyrakis, and R. Kopelman, Chem. Phys. Lett. 107, 48 (1984).

${ }^{4}$ P. Argyrakis and R. Kopelman, Phys. Rev. B 29, 511 (1984).

'S. Alexander and R. Orbach, J. Phys. Lett. 43, L625 (1985); R. Rammal and G. Toulouse, J. Phys. Lett. 44, L13 (1983).

'Y. Gefen, A. Aharony, and S. Alexander, Phys. Rev. Lett. 50, 77 (1983); P. G. de Gennes, C. R. Acad. Sci. Paris 296, 881 (1983); S. Havlin, D. BenAvraham, and H. Sompolinsky, Phys. Rev. A 27, 1730 (1983).

${ }^{7}$ Equation (4) is the correct scaling relation for $S_{N}$; however, in paper I, due to a typographical error the equation was given as

$$
S_{N}(t \rightarrow \infty, p)=t^{d / 2} f\left[\left(\frac{p}{p_{c}}-1\right) t-(2 v-p+\mu)\right] .
$$

${ }^{8}$ D. W. Heermann and D. Stauffer, Z. Phys. B 44, 339 (1981); R. B. Pandey and D. Stauffer, J. Phys. A 16, L511 (1983); J. Adler, Z. Phys. B 55, 227 (1984); D. S. Gaunt and M. G. Sykes, J. Phys. A 16, 783 (1983).

"A. A harony and D. Stauffer, Phys. Rev. Lett. 52, 2368 (1984\}.

${ }^{10}$ I. Webman, Phys. Rev. Lett. 52, 220 (1984).

"J. S. Newhouse, Ph.D. thesis, University of Michigan, Ann Arbor, 1985. 